\title{
Temporally distinct roles for tumor suppressor pathways in cell cycle arrest and cellular senescence in Cyclin D1-driven tumor
}

\author{
Hasan Zalzali ${ }^{1,2}$, Mohamad Harajly ${ }^{1}$, Lina Abdul-Latif ${ }^{1}$, Nader El-Chaar ${ }^{1}$, Ghassan Dbaibo $^{1}$, Stephen X Skapek ${ }^{3}$ \\ and Raya Saab ${ }^{1,2^{*}}$
}

\begin{abstract}
Background: Cellular senescence represents a tumor suppressive response to a variety of aberrant and oncogenic insults. We have previously described a transgenic mouse model of Cyclin D1-driven senescence in pineal cells that opposes tumor progression. We now attempted to define the molecular mechanisms leading to p53 activation in this model, and to identify effectors of Cyclin D1-induced senescence.

Results: Senescence evolved over a period of weeks, with initial hyperproliferation followed by cell cycle arrest due to ROS production leading to activation of a DNA damage response and the p53 pathway. Interestingly, cell cycle exit was associated with repression of the Cyclin-dependent kinase Cdk2. This was followed days later by formation of heterochromatin foci correlating with RB protein hypophosphorylation. In the absence of the Cdk4-inhibitor p18Ink4C, cell cycle exit was delayed but most cells eventually showed a senescent phenotype. However, tumors later arose from this premalignant, largely senescent lesion. We found that the p53 pathway was intact in tumors arising in a p18/nk4c-/- background, indicating that the two genes represent distinct tumor suppressor pathways. Upon tumor progression, both p18/nk4c-/- and p53-/- tumors showed increased Cdk2 expression. Inhibition of Cdk2 in cultured pre-tumorigenic and tumor cells of both backgrounds resulted in decreased proliferation and evidence of senescence.
\end{abstract}

Conclusion: Our findings indicate that the p53 and the RB pathways play temporally distinct roles in senescence induction in Cyclin D1-expressing cells, and that Cdk2 inhibition plays a role in tumor suppression, and may be a useful therapeutic target.

Keywords: p18Ink4c, Cyclin D1, Senescence, p53, Rb, Cdk2, Tumor, Reactive oxygen species

\section{Introduction}

Cellular senescence is a well-established tumor suppressor mechanism, activated in response to oncogenic signals, DNA damage, and telomere attrition among other protumorigenic insults (reviewed in [1,2]). Mechanistic insight into oncogene-induced senescence has emerged over the past few years. While it is clear that both the p53 and the $\mathrm{Rb}$ tumor suppressors are involved [3-6], their relative importance seems to vary depending on the activating insult

\footnotetext{
* Correspondence: rs88@aub.edu.lb

'Department of Pediatric and Adolescent Medicine, American University of Beirut, Beirut, Lebanon

${ }^{2}$ Children's Cancer Center of Lebanon, American University of Beirut, Beirut, Lebanon

Full list of author information is available at the end of the article
}

and cellular context [3,7-10]. Understanding the relative contributions of p53 and $\mathrm{Rb}$ to the induction and maintenance of senescence may have important implications, especially with development of targeted therapeutic agents.

Cyclin-dependent kinase 2 (Cdk2) was recently implicated in the senescence process, as Cdk2 loss was found to enhance senescence in Myc-induced tumors [11]. In addition, it was shown that Cdk2-dependent phosphorylation of Myc was necessary to bypass Ras-induced senescence [12]. This suggests that Cdk2 may act in senescence independently of its role in RB phosphorylation and cell cycle exit.

Here, we used a transgenic mouse model of premalignant Cyclin D1-driven pineal gland hyperplasia, to define the molecular mechanisms leading to p53 activation in

\section{Biomed Central}


response to Cyclin D1, and to identify effectors of Cyclin D1-induced senescence. The results shed light on the pattern of evolution of the senescence response in a premalignant lesion in vivo, and the differences in the contribution of the two major tumor suppressor pathways, p53 and RB. In addition, our findings suggest that Cdk2 inhibition may be a useful therapeutic approach, irrespective of the underlying genetic insult that led to senescence evasion.

\section{Results}

Cyclin D1-induced senescence in vivo occurs over a time frame of several weeks: We used the Irbp-Cyclin D1 mouse in which the expression of Cyclin D1 in the pineal gland causes excessive proliferation that is limited by senescence. The net result is a hyperplastic but senescent pineal gland that does not progress into an invasive tumor unless either $\mathrm{p} 53$ or the Cdk4-inhibitor $\mathrm{p} 18^{\mathrm{Ink} 4 \mathrm{c}}$ is lost [13]. We examined the temporal evolution of senescence and the contribution of the p53 and $\mathrm{Rb}$ tumor suppressor pathways to cell cycle exit in vivo. Histological studies of Cyclin D1-expressing pineal glands at various ages (post-natal day (P)10, P24, P35, and P49) showed that enhanced proliferation, measured by Ki67 immunostaining, was apparent at P10 but after this point it decreased such that essentially all cells had exited the cell cycle by P35 [Figure 1A, left panel, Quantitation in Figure 1B]. Cessation of proliferation preceded the formation of senescence-associated heterochromatin foci (SAHF); in fact, there was an unexpectedly long, two-week delay from P35 to P49 before SAHF were apparent [Figure $1 \mathrm{~A}$, middle and right panels]. Because SAHF have been observed in only a few mouse models of senescence [14-16], but not in other murine cells like MEFs [17], and because constitutive centromeric heterochromatin may show intense DAPI staining mimicking SAHF in normal murine cells $[18,19]$, we verified that these foci were indeed only seen in Cyclin D1-expressing cells and not the wild-type counterparts at P49 [Additional file 1: Figure S1A]. We could not detect positive staining for senescence-associated beta galactosidase (SABG), another marker of senescence [20]. However, we believe this must be a technical issue (detecting the enzyme activity necessitates freshly frozen tissue), because when the Cyclin D1expressing pineal cells were grown in vitro, they showed features of senescence that included positive staining for SABG [see below, and Additional file 1: Figure S1B]. In addition, we evaluated the pineal cells for other known markers of senescence, such as Dec1, DcR2, and p15Ink4b [21]. We found that, concomitant with cell cycle exit and SAHF formation, all three markers of senescence were increased at P49 [Figure 1C]. We conclude that Cyclin D1-induced senescence in the pineal cells occurs by P49, and that cell cycle exit in this setting occurs many days prior to expression of bona fide markers of senescence.

p53 activation occurs prior to cell cycle exit, and is mediated by a ROS-induced DNA damage response: To identify when p53 was engaged in tumor suppression, we

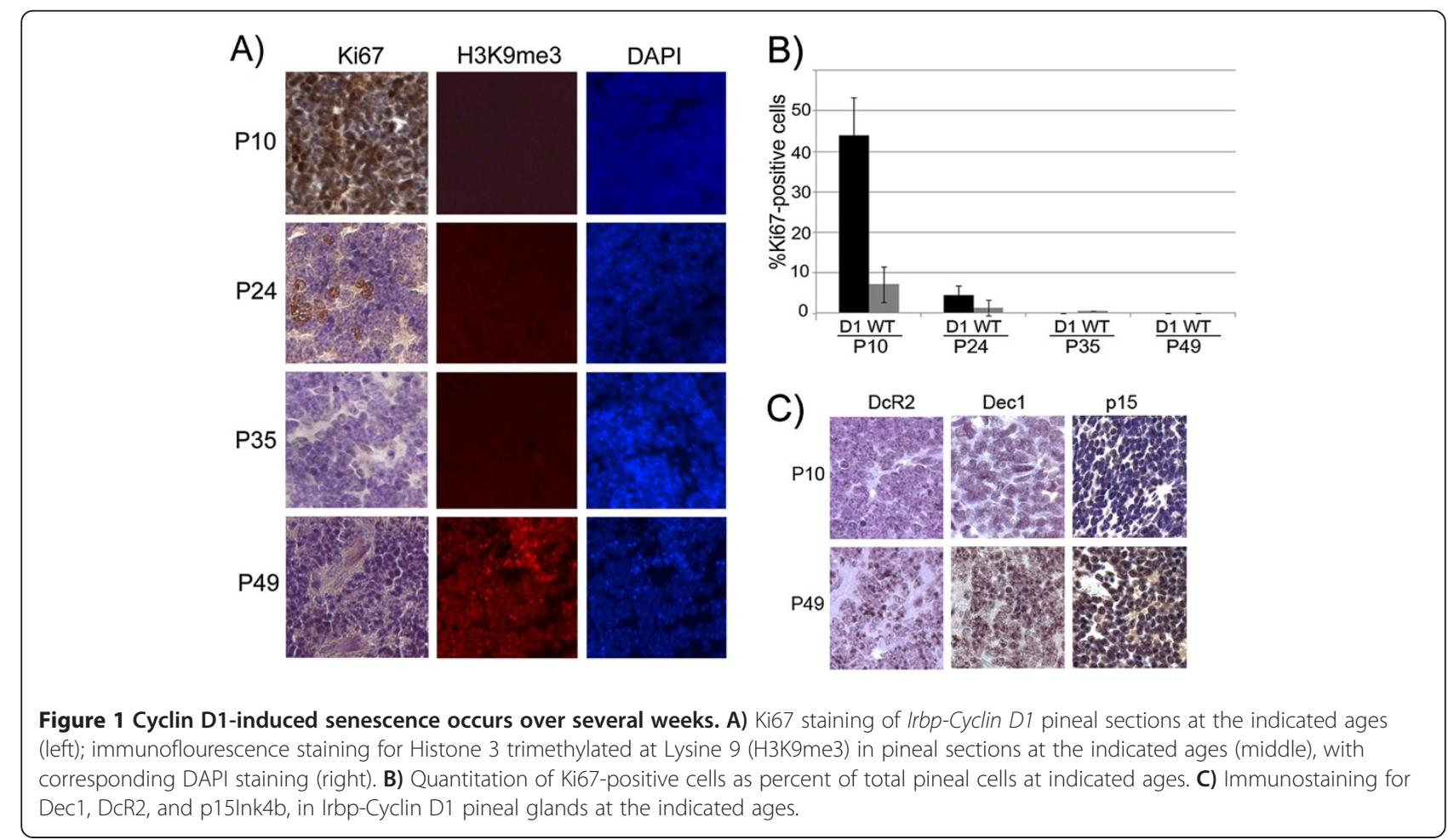


evaluated cell lysates of pineal glands collected at different postnatal ages. We found that at P10, p53 protein expression was increased, as was its phosphorylation at Ser15/20 (a site phosphorylated by the DNA damage response) [Figure 2A]. The p53 target, p21 ${ }^{\text {Cip } 1}$, was also induced at that time-point [Figure 2A]. However, p53 activation did not persist once the cells had exited the cell cycle nor was it detectable at P49 when the cells displayed SAHF [Figure 2A].

We hypothesized that Cyclin D1 expression may be inducing p53 through activation of the DNA damage response (DDR), as reported for Ras-induced senescence (reviewed in [22]). Indeed, we found nuclear accumulation of phosphorylated histone H2AX (pH2AX) at P10, concomitant with p53 activation [Figure 2B, $2 \mathrm{C}$. In addition, Chk1 was phosphorylated concomitantly with phosphorylation of $\mathrm{p} 53$, further indicating that the DDR pathway was active in the transgenic pineal gland at this time, but not at later time-points [Figure 2D]. These findings reveal that deregulated Cyclin D1 enhances the DDR pathway and activates p53 while cells are proliferating, but ongoing DDR and active p53 are not needed after cells have undergone senescence.

We next assessed whether reactive oxygen species (ROS) may be contributing to Cyclin D1-induced senescence in pineal cells, based on their role in Ras-induced senescence in cultured fibroblasts [23-25]. Using DCFDA assay in explanted pineal cells, we found that ROS were indeed induced in response to Cyclin D1 expression, but not in wild-type pineal cells grown in the same conditions [Figure 3A]. We investigated whether ROS were responsible for activation of the DNA damage response in this setting. Indeed, Cyclin D1-induced ROS resulted in DNA damage foci marked by $\mathrm{pH} 2 \mathrm{AX}$, as well as the ROS-induced incorporation of oxidized dNTP, 8Oxo-dGTP, into DNA [Additional file 1: Figure S1D]. It also led to phosphorylation of Chk1, a component of the DNA damage response pathway [Figure 3B, left panel], and to increased expression of two p53 pathway effector proteins, 14-3-3 and p21 [Figure 3B, middle and right panels]. Treating cells with the ROS scavenger N-Acetyl Cysteine (NAC) resulted in abrogation of DNA damage [Additional file 1: Figure S1D], abrogation of DDR

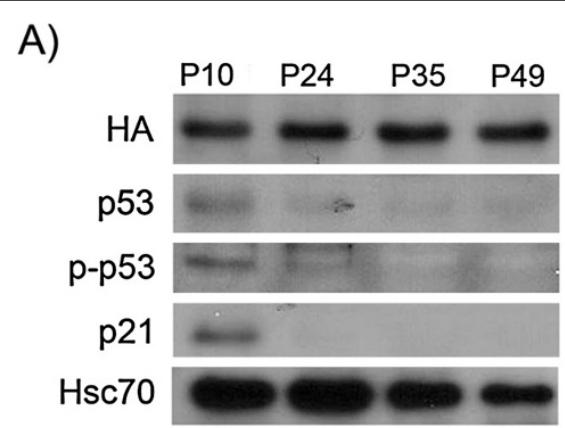

C)

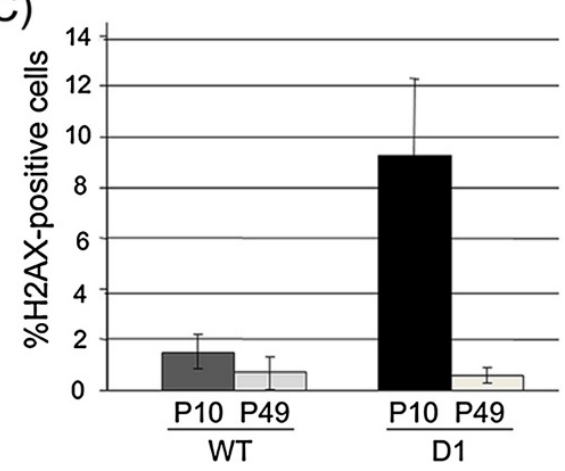

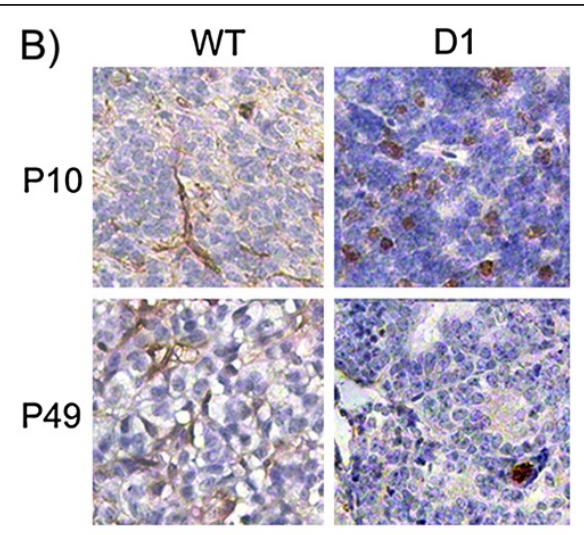

D)

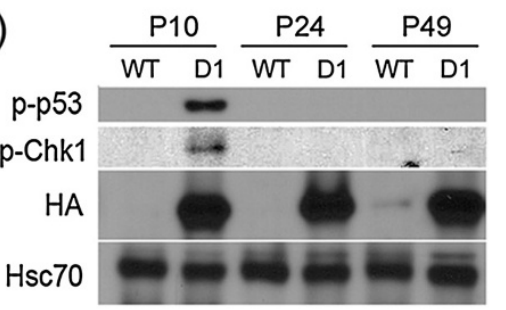

Figure 2 Cyclin D1-induced cell cycle exit is associated with early p53 activation and a DNA damage response. A) Western blotting for the indicated proteins in Irbp-Cyclin D1 pineal glands at the indicated ages. HA: HA epitope on the Cyclin D1 transgene. B) Representative immunostaining for $\mathrm{yH} 2 \mathrm{AX}$ in Irbp-Cyclin D1 (D1) and wild-type (WT) pineal sections at the indicated ages. C) Quantitative analysis of $\gamma \mathrm{H} 2 \mathrm{AX}$ -positive pinealocytes in wild-type (WT) and Irbp-Cyclin D1 (D1) pineal sections at the indicated ages. Columns: mean of measurements from three to six separate mice; bars, SD. D) Western blotting for the indicated proteins in wild-type (WT) and Irbp-Cyclin D1 (D1) pineal glands at the indicated ages. HA: HA epitope on the Cyclin D1 transgene. Hsc70 is a loading control. 


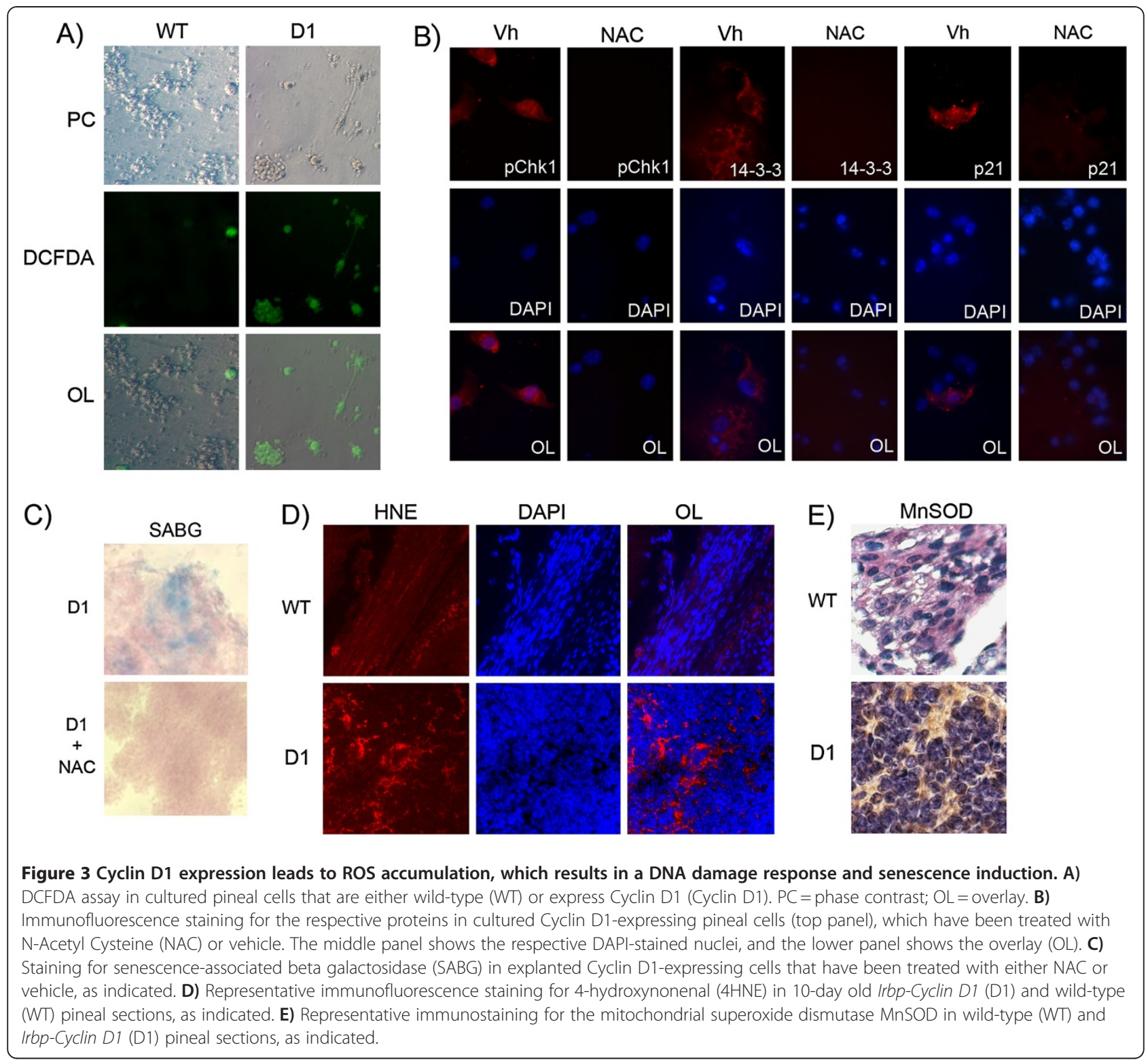

activation, and absence of p53 pathway activation [Figure 3B], as well as evasion of senescence [Figure 3C]. By staining for 4-hydroxy-nonenal (HNE), a marker of lipid oxidation, we found evidence of oxidative stress in pineal sections of Irbp-Cyclin D1 mice, but not wild-type mice [Figure 3D], and there was also increased expression of the mitochondrial superoxide dismutase protein MnSOD [Figure 3E], which is induced by ROS stress in vivo [26]. Thus, from the above in vitro and in vivo evidence, we conclude that Cyclin D1 expression results in accumulation of ROS, which in turn leads to activation of the DDR and the p53 pathway, resulting in induction of senescence.

$\underline{\mathrm{RB} \text { pathway activation occurs after cell cycle exit and }}$ is associated with SAHF: To evaluate when the $\mathrm{Rb}$ pathway was engaged, we used western blotting to investigate the Cdk-dependent phosphorylation of $\mathrm{Rb}$ in pineal cell lysates. We investigated the status of $\mathrm{Rb}$ phosphorylation at Cdk4-dependent sites such as Ser790, and at Cdk2-dependent sites such as Ser612 [Figure 4A]. We found that $\mathrm{Rb}$ was phosphorylated at Cdk2dependent sites (Ser612) at P10, when cells were proliferating, but decreased after cells exited the cell cycle by P24 [Figure 4A]. In contrast, Rb was phosphorylated at Cdk4-dependent sites (Ser790) from P10 through P35, even though most cells had ceased to proliferate by P24 [Figure 4A]. Rb phosphorylation at Cdk4-dependent sites was reversed at P49 as SAHF formed [Figure 4A].

To understand the mechanism of $\mathrm{Rb}$ activation, we investigated the expression of the Cdk-inhibitors p16Ink4a, 

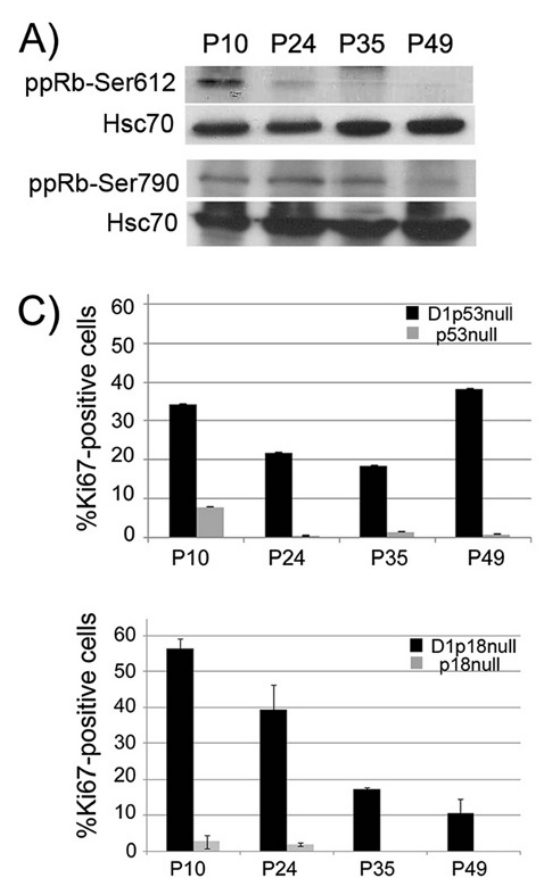

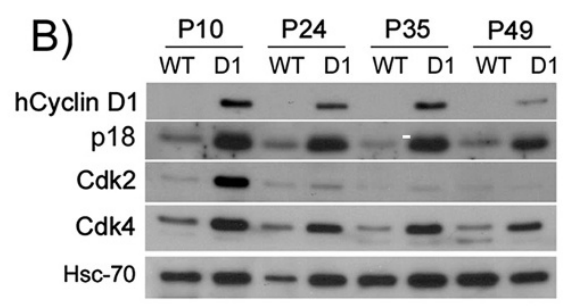

D) $\frac{10 d}{D 1 D 1 p 18 n} \frac{24 d}{D 1 \quad D 1 p 18 n} \frac{49 d}{D 1 \quad D 1 p 18 n}$

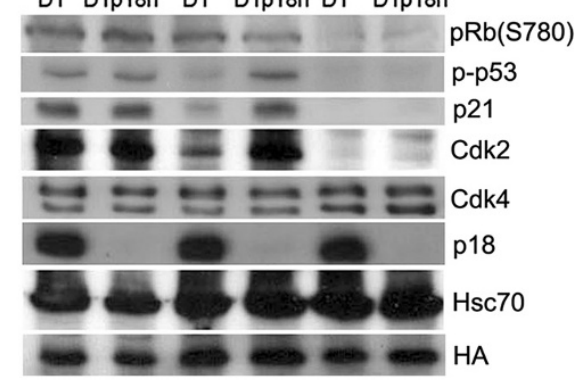

Figure 4 p18Ink4c loss delays Cyclin D1-induced senescence. A) and B) Western blotting for the indicated proteins in wild-type (WT) and IrbpCyclin D1 (D1) pineal glands at the indicated ages. Hsc70 is a loading control. C) Quantitative analysis of Ki67-positive pinealocytes in Irbp-Cyclin D1, p53-/ (D1p53n), and Irbp-Cyclin D1, p18Ink4c-/- (D1p18n) mice of the indicated ages, compared to p53-/- and p18/nk4c-/-controls, respectively. Columns: mean of measurements from three to six separate mice; bars, SD. D) Western blotting for the indicated proteins in Irbp-Cyclin D1 (D1) and Irbp-Cyclin D1, p18Ink4c-/- (D1p18n) pineal glands at the indicated ages. HA: epitope on the Cyclin D1 transgene.

p15Ink4b, p18Ink4c, and p27Kip1 [27-30]. There was increased expression of $\mathrm{p} 18^{\text {Ink4c }}$ at all time points [Figure 3B], an increase in p15 ${ }^{\text {Ink4b }}$ expression at P49 [see Figure 1D], but no changes in expression of $\mathrm{p} 16^{\text {Ink4a }}$ and p27 ${ }^{\text {Kip1 }}$ (not shown). We also evaluated the expression of Cdk4 and Cdk2, especially since Cdk2 inhibition was recently found to be important for Myc-induced senescence [11]. We observed a modest decrease in Cdk4 expression from P10 through P49 [Figure 3B], but interestingly we found that Cdk2 expression was markedly reduced from P10 to P24 [Figure 3B], coincident with the timing of cell proliferation arrest and loss of $\mathrm{Rb}$ phosphorylation at Cdk2-specific sites. We conclude that Cdk2 repression correlates most closely with the initial proliferation arrest; and that diminished Cdk4-dependent Rb phosphorylation occurs at a later time-point and correlates with formation of SAHF.

p18 ${ }^{\text {Ink4c }}$ loss delays p53-dependent cell cycle exit: Because of the observed increase in expression of p18 ${ }^{\text {Ink4c }}$, we used a genetic approach to evaluate its role in Cyclin D1-induced senescence. In contrast to what occurs without p53 - where cell proliferation only slightly decreases from P10 to P35 and then increases as invasive tumor progresses [Figure 4C, top panel; and [13]], proliferation decreased from P10 through P49 without p18Ink4c
[Figure 4C, bottom panel], but exceeded that in the IrbpCyclin D1 cells [Figure 4C, bottom panel, compare with Figure 1B].

Notably, p53 activation measured by phosphorylation at Ser15/20, and expression of the p53-target $\mathrm{p} 21^{\mathrm{Cip} 1}$, persisted until P24 in Irbp-Cyclin D1, p18Ink4c -/- cells [Figure 4D], correlating with the prolonged cellular proliferation. Further, Cdk2 expression persisted until P24 in Irbp-Cyclin D1, p18Ink4c -/- cells [Figure 4D]. These findings indicate that $p 18 \operatorname{Ink} 4 c$ loss delayed but did not prevent p53-dependent events leading to cell cycle exit.

Interestingly, loss of Cdk4-dependent $\mathrm{Rb}$ phosphorylation still occurred in the absence of $p 18 \operatorname{Ink} 4 c$, again correlating with the appearance of SAHF [Figure 4D]. In fact, the majority of Irbp-Cyclin D1, p18Ink4c -/- cells displayed SAHF by P49 [Additional file 2: Figure S2A], whereas SAHF never formed in Irbp-Cyclin D1, p53 -/cells [13]. In addition to SAHF, the senescence markers Dec1 and DcR2 were also expressed in Irbp-Cyclin D1, p18Ink4c - /- cells at P49 [Additional file 1: Figure S2B]. Findings were similar in vitro using pineal cells explanted from P10 animals and cultivated for 10-20 days: Explanted Irbp-Cyclin D1 cells showed evidence of senescence, including loss of proliferation (measured by BrdU incorporation), and positive staining for SABG, by 
10 days in culture [Additional file 1: Figure S1B, 1C], while the Irbp-Cyclin D1, p53 -/- cells continued to proliferate and did not senesce [Additional file 1: Figure $\mathrm{S} 1 \mathrm{~B}$, bottom]. In contrast, the Irbp-Cyclin D1, p18Ink4c -/- cells did show evidence of senescence, but it was delayed until close to 20 days in culture [Additional file 2: Figure S2C]. We conclude that $\mathrm{p} 18^{\text {Ink } 4 \mathrm{c}}$ slowed proliferation but was not essential for most Cyclin D1 expressing cells to cease proliferating and become senescent.

p53 and p18 $8^{\text {Ink4c }}$ act independently in suppressing Cyclin D1-driven tumors: The persistence of a small number of proliferating cells by $\mathrm{P} 49$, in Irbp-Cyclin D1, p18Ink4c - / - mice, was important because it led to pineoblastoma by 7-10 months of age in all mice examined $(n=15)$. Examining mice at 3-5 months of age, we observed an obvious border between the malignant and pre-malignant parts of the pineal gland [Figure 5A, left]; this border disappeared as the tumor infiltrated the whole pineal gland [Figure 5A, right], and SAHF were lost in the emerging Irbp-Cyclin D1, p18Ink4c -/- tumor [Figure 5B]. Dual immunostaining for BrdU and SAHF clearly demonstrated that proliferating pinealocytes were distinct from those that displayed SAHF [Figure 5C].

We considered whether the prolonged proliferation in the absence of $\mathrm{p} 18^{\text {Ink4c }}$ might have derailed a p53dependent arrest in the malignant tumors. Western blotting showed that Irbp-Cyclin D1, p18Ink4c -/- tumors still expressed the p53 protein [Figure $5 \mathrm{D}$ ], and sequencing of p53 exons 5-8 did not reveal mutations in genomic DNA from nine different Irbp-Cyclin D1, p18Ink4c -/- pineal tumors (data not shown). Further, using primary cultures of pineal tumor cells, we found that both gamma irradiation and treatment with etoposide resulted in increased p53 phosphorylation and in p53-dependent increases in $\mathrm{p} 21^{\mathrm{Cip} 1}$ and 14-3-3 in Irbp-Cyclin D1, p18Ink4c -/- but not Irbp-Cyclin D1, p53 -/- tumor cells
A)

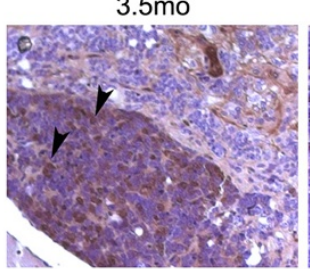

B)

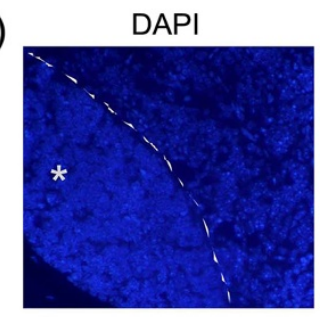

C)

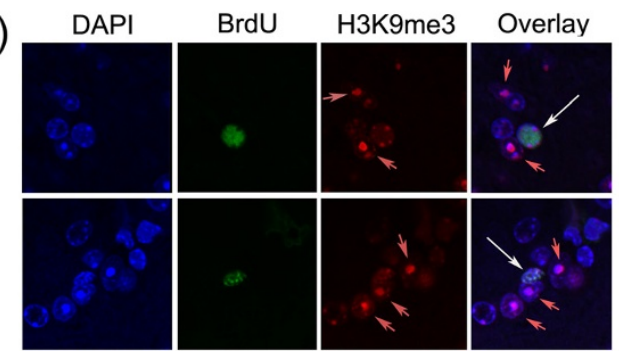

H3K9me3

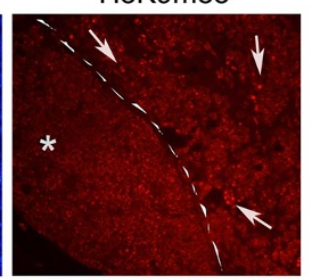

\section{E)}
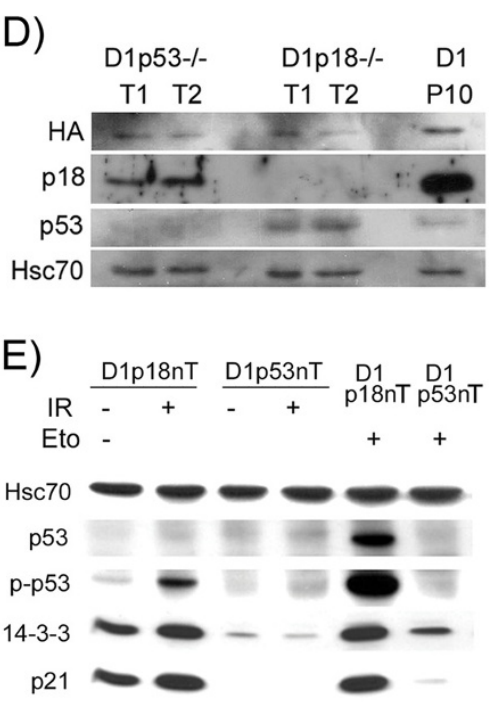

F)

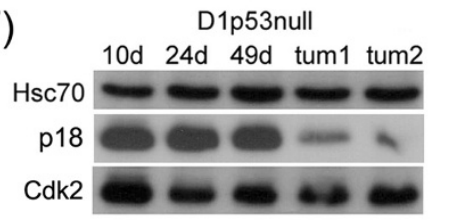

Figure 5 p18Ink4c loss promotes emergence of tumor from a premalignant senescent lesion. A) Representative pineal sections of IrbpCyclin D1, p181nk4c-/- mice at the indicated ages, with Ki67 immunostaining. Arrowheads mark examples of Ki67-positive cells. B) Immunoflourescence for H3K9me3 and corresponding DAPI staining of Irbp-Cyclin D1, p18Ink4C-/- pineal sections at 3.5 months. Asterisk denotes the emerging tumor; dashed line demarcates tumor from the senescent pineal lesion. Arrows indicate examples of cells with SAHF (positive for H3K9me3). C) Dual immunoflourescence for BrdU (green) and H3K9me3 (red) in Irbp-Cyclin D1, Ink4c-/- pineal section at P49. Red arrows indicate cells with H3K9me3 foci, white arrows cells positive for BrdU. D) Western blotting for the indicated proteins in representative tumors from IrbpCyclin D1, p53-/- (D1p53n) and Irbp-Cyclin D1, p18Ink4c-/- (D1p18n) mice, compared to a P10 Irbp-Cyclin D1 pineal gland (D1). HA: HA tag of the Cyclin D1 transgene. E) Representative western blot for the indicated proteins in tumor cells from Irbp-Cyclin D1, p53 -/- (D1p53nT) and Irbp-Cyclin D1, p18/nk4C -/- (D1p18nT) mice, before and after treatment with ionizing radiation or etoposide, as indicated. F) Western blotting for the indicated proteins in Irbp-Cyclin D1, p53 -/- (D1 p53null) pineal lysates at the indicated ages and in tumor lysates as indicated. 
[Figure 5E]. These findings confirmed that p53 remained intact in Irbp-Cyclin D1, p18Ink4c -/- tumor cells. In contrast, there was decreased $\mathrm{p} 18^{\text {Ink4c }}$ expression in Irbp-Cyclin D1, p53-/- tumors, suggesting that p18 may act as a tumor suppressor, even in a p53-null setting [Figure 5D, 5F]. However, preliminary results show no enhanced tumor susceptibility in Irbp-Cyclin D1, p53-/-, p18Ink4c -/- (double knock-out) animals (data not shown).

Cdk2 is induced in both p18Ink4c - / and p53 -/tumors, and may be a suitable therapeutic target: Several changes in Cdk2 expression suggested that it may represent a critical effector of Cyclin D1-driven tumorigenesis. In the Irbp-Cyclin D1, and Irbp-Cyclin D1, p18Ink4c-/animals, Cdk2 was repressed as cells ceased to proliferate, and repression was markedly blunted in the IrbpCyclin D1, p53 -/- pineal gland [Figure 5F, compare with Figure 4D], in which neither cell cycle arrest nor senescence was observed [see Figure 4A]. Repression of Cdk2 seemed specific because there was no repression of another closely related cell cycle protein, Cdk1 [Additional file 2: Figure S2D]. Lastly, Cdk2 increased in tumors progressing from the largely senescent, Irbp-Cyclin D1, p18Ink4c -/- pineal gland [Figure 6A], and Cdk2 expression correlated with Ki67-positivity in emerging tumors [Figure 6B]. Areas in the Irbp-Cyclin D1, p18Ink4c -/tumors that remained Ki67-negative displayed little Cdk2 [Figure 6B and 6C].

To address the role of $\mathrm{Cdk} 2$ repression as a possible therapeutic target, we treated explanted Irbp-Cyclin D1, p18Ink4c -/- and Irbp-Cyclin D1, p53 -/- pineal tumor cells with the Cdk2-inhibitor CVT313, at a concentration of $5 \mu \mathrm{M}$, known to specifically inhibit Cdk2 [11,31]. CVT313 treatment decreased cell number (estimated by total cellular area) in Irbp-Cyclin D1, p18Ink4c -/- and Irbp-Cyclin D1, p53 -/- tumor cells in 8-well chamber slides [Figure 6D, upper panel]. Furthermore, CVT313-treated cells showed an increase in positive staining for SABG activity [Figure 6D, lower panel, and Figure 6E]. Importantly, treatment of pretumorigenic Irbp-Cyclin D1 pineal cells with CVT313 also decreased the apparent cell number [Figure 6E], while treatment of wild-type pineal cells did not seem to have a noticeable effect, either on cellularity or SABG positivity [Figure 6E].

We assessed whether Cdk2 inhibition by CVT313 was primarily affecting cellular proliferation by BrdU incorporation assay. Indeed, we found that CVT313 treatment decreased proliferation in oncogene-expressing and pretumorigenic cells, but not in wild-type pineal cells [Figure 7A, quantitation in Figure 7B]. There was no evidence of any increase in apoptotic cells after CVT313 treatment in either cell type, as measured by TUNEL staining (negative data not shown).
To investigate whether the effects on senescence induction were specific to Cdk2 inhibition, we treated explanted Irbp-Cyclin D1, p53-/- and Irbp-Cyclin D1, p18Ink4c-/- cells with a specific Cdk4-inhibitor, NSC 625987 [32,33]. Inhibition of Cdk4 decreased proliferation (measured by BrdU incorporation), though to a lesser extent than seen with Cdk2 inhibition [Figure 7C]. However, unlike Cdk2 inhibition, it did not result in any detectable increase in SABG staining [Figure 7D]. This demonstrates that Cdk2 inhibition was specifically relevant to induction of senescence in Cyclin D1-expressing pineal cells.

\section{Discussion}

Emerging evidence supports the concept that cellular senescence represents a likely mechanism through which oncogenic transformation is suppressed. Senescence has been observed in pre-malignant lesions in mouse and in man, but not in fully transformed counterparts of these lesions (reviewed in [21,34]). Because of the time lag in progression of premalignant lesions and the incomplete penetrance, it has been assumed that accumulation of as yet poorly-defined genetic or epigenetic changes likely contribute to the emergence of a tumor from a premalignant, apparently senescent lesion.

Our work provides new insight into cellular and molecular events that occur as oncogene-expressing cells arrest, become senescent, and finally emerge or escape from the senescent state as a malignant tumor. This is the first in vivo description of temporal morphologic and molecular events accompanying the evolution of an oncogene-driven senescent state, showing a previously unrecognized temporal sequence where cell cycle exit preceded formation of heterochromatin foci by several weeks.

Two tumor suppressor genes, $p 53$ and $p 18 \operatorname{Ink} 4 c$, played distinct roles during this process. P53 activation occurred concomitantly with an active DNA damage response, and was essential to drive cell cycle exit, temporally associated with Cdk2 repression and loss of Cdk2-dependent phosphorylation of the retinoblastoma protein Rb. Days later, reversal of Cdk4-dependent phosphorylation of the $\mathrm{Rb}$ protein correlated with the emergence of morphological and biochemical changes of oncogene-induced senescence. At that point, though, there was no evidence of p53 pathway activation. This is the first direct in vivo evidence for distinct temporal roles for these two tumor suppressors in the senescence process.

The early and transient activation of the p53 pathway suggested that p53 was integral for the initial cell cycle exit but not directly involved in formation of SAHF. Other models have also shown conflicting and contextdependent evidence for the role of $\mathrm{p} 53$ in the formation 


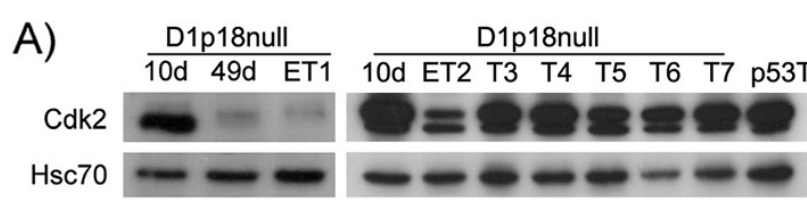

B)

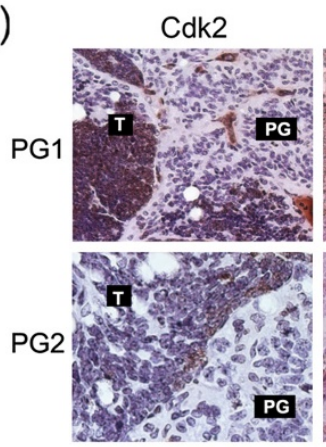

D)
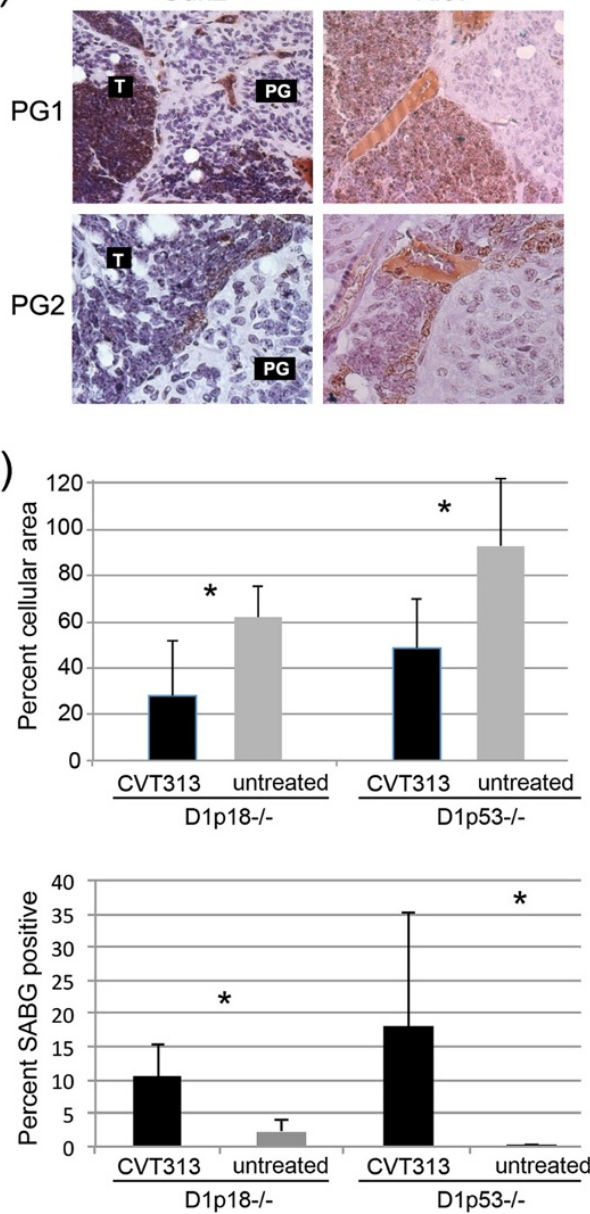

C)
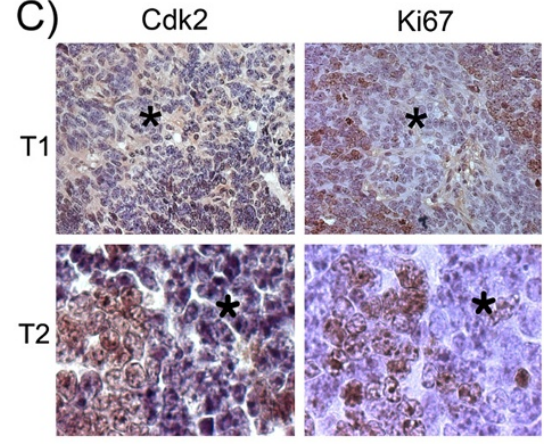

E)
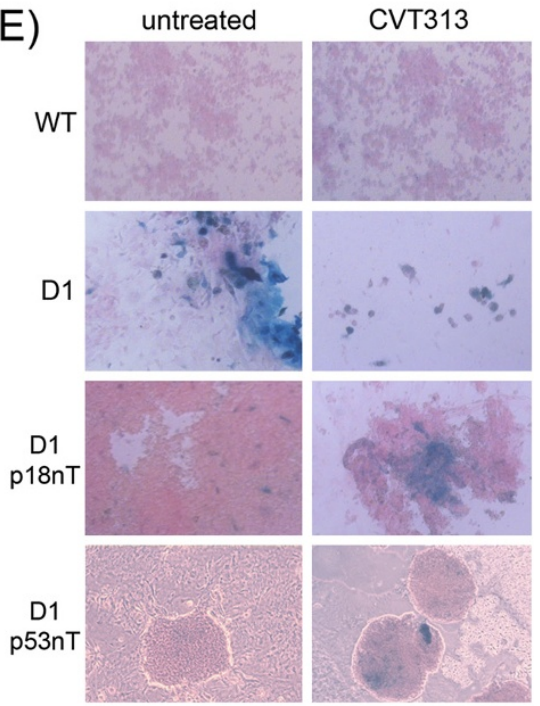

Figure $\mathbf{6}$ Cdk2 is a therapeutic target in tumors that have bypassed senescence. A) Western blotting for the indicated proteins in Irbp-Cyclin D1, p181nk4C \% (D1p18null) pineal lysates at the indicated ages, Irbp-Cyclin D1, p181nk4C - (D1p18null) tumors that are early (ET1, ET2) or wellestablished (T3 - T7), and in Irbp-Cyclin D1, p53 - - (p53T) tumor lysates, as indicated. B) Representative immunohistochemical staining for Cdk2 and Ki67, as indicated, in serial sections of Irbp-Cyclin D1, p18/nk4C - - pineal glands (PG1 and PG2) that have an emerging tumor within a senescent background. "T" denotes the emerging tumor; "PG" denotes the pineal gland background. C) Representative immunohistochemical staining for Cdk2 and Ki67, as indicated, in serial sections of Irbp-Cyclin D1, p18Ink4C - - tumors (T1 and T2), showing areas of low Ki67 and low Cdk2 positivity (asterisks). D) Quantitation of cellular accumulation (upper panel) and SABG-positive proportion (lower panel) of Irbp-Cyclin D1, p18Ink4C - - and Irbp-Cyclin D1, p53 - - tumor cells after treatment for 7 days with CVT313 or vehicle, as indicated. Asterisk denotes statistical significance ( $p$-value $=2.7 \times 10^{-3}, 9.8 \times 10^{-4}$ respectively, for the upper panel; $6.4 \times 10^{-4}$ and $6.3 \times 10^{-4}$, respectively for the lower panel). E) Representative SABG staining of wild-type (WT, upper panel), pretumorigenic Irbp-Cyclin D1 (D1, second panel), Irbp-Cyclin D1, p18Ink4C -/(D1p18n, $3^{\text {rd }}$ panel) and Irbp-Cyclin D1, p53 - - (D1 p53n, lower panel) tumor cells in culture, treated for 7 days with CVT313 or vehicle, as indicated.

of SAHF [35-37]. In contrast, Rb activation was delayed and stable. Rb seemed to be important in both cell cycle exit as well as formation of SAHF: compromise of the $\mathrm{Rb}$ pathway through loss of p18Ink4c (the major known function of which is inhibition of $\mathrm{Cdk} 4 / 6$ to indirectly activate $\mathrm{Rb}$ [38-40]) led to a delay in initial cell cycle exit, and eventually to complete penetrance of tumor progression within the senescent-like lesion. Taken together, these findings implicate $\mathrm{Rb}$, rather than $\mathrm{p} 53$, as the key protein needed to foster the emergence and maintenance of SAHF, thought to be responsible for repression of cell-cycle genes [3,35]. Involvement of $\mathrm{Rb}$ in the 


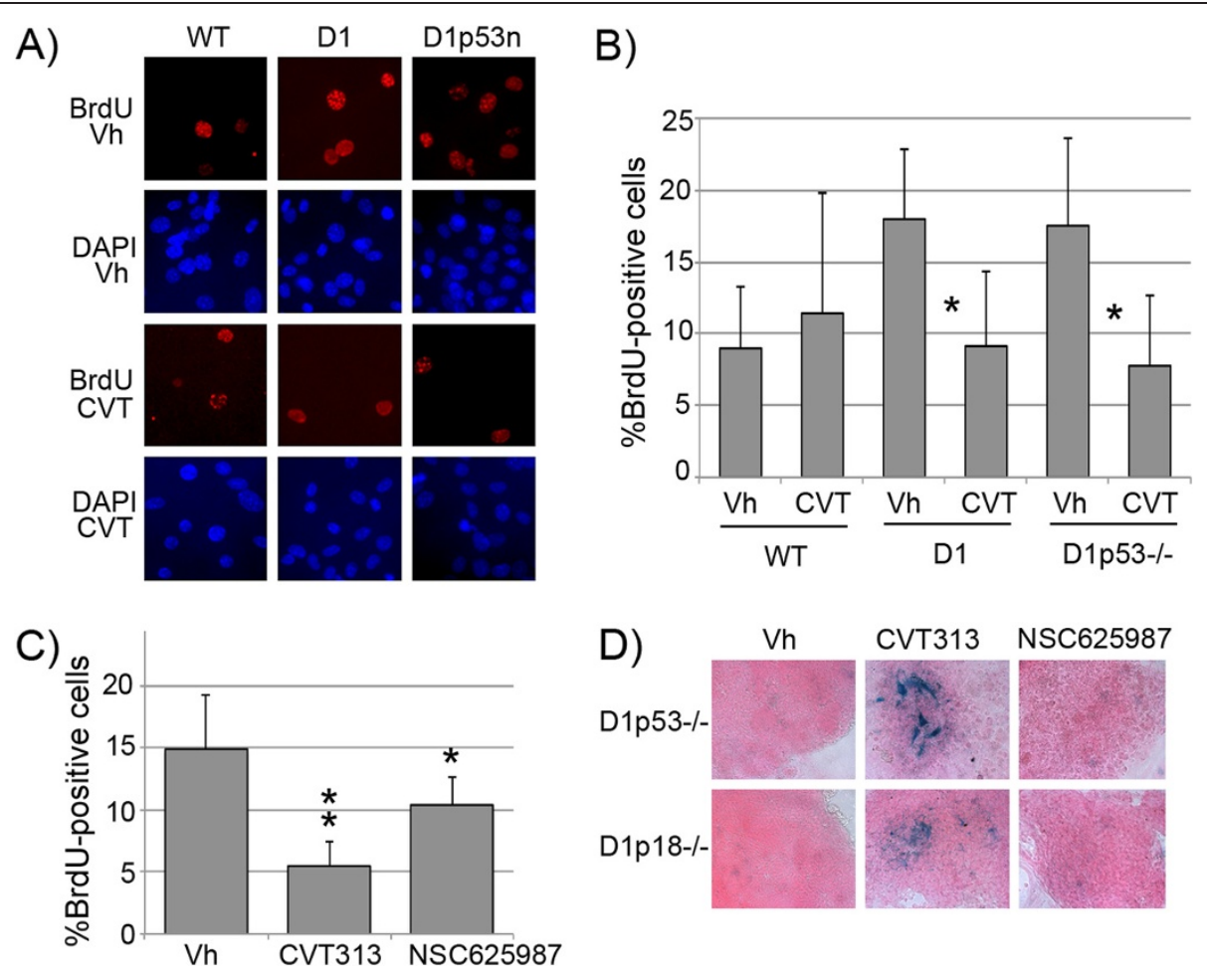

Figure 7 Cdk2 inhibition in tumor cells leads to features of senescence. A) BrdU-incorporation and immunofluorescence staining (red), and corresponding DAPI staining (blue), of cultured wild-type (WT), Irbp-Cyclin D1 (D1), and Irbp-Cyclin D1,p53-/- (D1 p53-/-) pineal cells, as indicated, after 7 days of treatment with CVT313 (CVT) or an equal volume of DMSO vehicle (Vh). B) Quantitative analysis of percent BrdU-positive cells in wild-type (WT) and Irbp-Cyclin D1 (D1), and Irbp-Cyclin D1,p53-/- (D1 p53-/-) pineal cells treated for 7 days with CVT313 (CVT) or vehicle (Vh). Asterisk denotes statistical significance. C) Quantitative analysis of percent BrdU-positive cells in Irbp-Cyclin D1,p18/nk4c-/-tumor cells treated for 7 days with CVT313 (CVT), NSC625987, or vehicle (Vh). Asterisk denotes statistical significance. Double asterisk is for statistically significant decrease in comparison to both other conditions. D) Representative SABG staining of Irbp-Cyclin D1, p53 -/- (D1p53-/-) and Irbp-Cyclin D1, p18Ink4C -/(D1 p18-/-) tumor cells in culture, treated for 7 days with CVT313, NSC625987, or vehicle, as indicated.

formation of SAHF has been shown in other settings: human RB was shown to directly co-localize with SAHF $[35,36]$, and inactivation of the $\mathrm{p} 16 \mathrm{INK} 4 \mathrm{a} / \mathrm{RB}$ pathway impairs formation of RasG12V-induced SAHF in human fibroblasts [35]. In our model, p53-driven cell cycle exit correlated with hypo-phosphorylation of $\mathrm{Rb}$ at $\mathrm{Cdk2-}$ dependent sites, while formation of SAHF correlated with hypo-phosphorylation at Cdk4-dependent sites. This suggests that hypo-phosphorylation of these specific residues may be involved in SAHF formation. It will be interesting to evaluate whether mutated forms of $\mathrm{Rb}$ that cannot be phosphorylated at these particular Cdk4 sites can more robustly foster the appearance of SAHF.

Our results suggest that $p 53$ and $p 18 \operatorname{Ink} 4 c$ represent separate tumor suppressor pathways in Cyclin D1-driven pineoblastoma. While tumors progressed within 34 months in p53 -/- animals, they appeared much later, after 7-10 months in $p 18 \operatorname{Ink} 4 c-/-$ mice. Also, the p53 pathway was intact in $p 18 \operatorname{In} k 4 c-/$ - tumors, further proving that the two pathways of tumor suppression are distinct. Tumor suppression required functional $p 53$ and
p18Ink4c, as neither was sufficient to prevent tumor progression alone. While the cell cycle exit after P10 was clearly p53-dependent, absence of p18Ink4c delayed the cell cycle exit but did not prevent it in the majority of cells, which went on to express other markers of senescence. However, few cells continued to proliferate, resulting in tumorigenesis. It thus appears that, while p53 loss resulted in abrogation of cell cycle exit altogether, loss of $\mathrm{p} 18^{\text {Ink4c }}$ decreased the threshold for bypass of the $\mathrm{p} 53$ dependent cell cycle exit in a subset of cells.

In our model, p53-dependent cell cycle arrest was associated with marked Cdk2 repression, while Cdk2 levels were maintained in Irbp-Cyclin D1, p53 -/- cells which never exited the cell cycle [see Figure 5F]. While a role for $\mathrm{Cdk} 2$ repression in facilitating senescence has been shown in an earlier report [11], ours is the first description of Cdk2 repression occurring in a temporal association with p53-dependent cell cycle exit. This indicates that Cdk2 repression might be a novel p53-dependent mechanism to foster cell cycle exit, especially since no similar changes were seen in the related cell cycle 
regulator Cdk1. However, additional work will be needed to investigate whether Cdk2 repression is a direct p53dependent effect, and whether it is sufficient to induce cell cycle exit and induction of senescence. Moreover, since Cdk2 and other Cdks are also regulated post-transcriptionally by phosphorylation and by their binding to CDK-inhibitors, future work should focus on elucidating these molecular aspects for complete mechanistic understanding of the role of Cdk2 and other Cdks in inducing senescence. Future studies utilizing the established in vitro model, as well as genetically engineered mouse models, should be able to specifically dissect the role of Cdk2 in tumor progression, and upstream and downstream mechanisms leading to its repression and to cell cycle exit.

Finally, although tumors arising in a $p 53$-/- setting were molecularly different from those arising in a p18Ink4c -/- setting, Cdk2 levels were high in both (compared to senescent cells) and both cell types responded to Cdk2 inhibition. While Cdk4 inhibition also decreased cell proliferation, only Cdk2 inhibition resulted in features of senescence in treated cells. These conclusions are based on the published specificity of the inhibitors used at the corresponding concentrations. Ideally, we would have preferred to document inhibitor-induced $\mathrm{pRb}$ specific phosphorylations and specific kinase activity in each experiment, however the low number of primary cells used in these experiments was prohibitive.

Keeping the above limitations in mind, these findings provide a rationale for exploring the use of pharmacological Cdk inhibition, specifically Cdk2, to induce senescence in tumor cells, irrespective of whether the p53 pathway is compromised. Such an approach to therapy may be especially useful in tumors where the primary insult lies with deregulated Cyclin D1 expression, as in the reported model.

\section{Materials and methods Mouse Studies}

Irbp-Cyclin D1 transgenic mice [41] were bred with $p 53$ -/- (Jackson Laboratory, Maine), or p18Ink4c -/- mice [42] and maintained in a mixed C57BL/ $6 \times 129 /$ Sv genetic background. PCR for targeted alleles was used to verify mouse genotypes as described [41,42]. Animals were euthanized at defined time points or when obviously ill in accordance with the American University of Beirut (AUB) Institutional Animal Care and Use Committee guidelines; all studies were approved by this committee.

\section{Analyses of protein expression}

Protein lysates were prepared from pineal tissue by lysis in Universal Lysis Buffer. Electrophoresis was performed using 8,10 , or $12 \%$ Tris-Chloride gels, transferred to polyvinylidene difluoride membranes (Bio-Rad
Laboratories, Hercules, CA), and detected using antibodies to p21 ${ }^{\text {Cip1 }}$, Cdk4, Cdk2, Cdk1, Hsc70, phosphoS790 Rb, total and phospho-specific p53 at Ser15/20 (Santa Cruz Biotechnology, Santa Cruz, CA); phosphoS612 Rb (MBL International, Woburn, MA); p18 ${ }^{\text {Ink4c }}$ (Zymed, San Francisco, CA); human Cyclin D1 (BDPharmingen, San Diego, CA); hemagglutinin (HA) epitope (Covance, Trenton, NJ); and 14-3-3 (Abcam, Cambridge, UK).

For evaluation of p53 pathway, pineal tumors were excised, dispersed, and plated onto permanox chamber slides, and grown in culture in 10\% FBS in DMEM for 72 hours, then treated with 6 Gy irradiation at $1 \mathrm{~Gy} / \mathrm{min}$, or $10 \mu \mathrm{M}$ Etoposide. Cells were collected 24 hours after irradiation, or 6 hours after Etoposide treatment, and protein extraction and western blotting was performed as above.

\section{Histological studies and Immunostaining}

Brain tissue was fixed in $4 \%$ paraformaldehyde for 72 hours then embedded in paraffin. For mice older than 10 days, skulls were peeled off before embedding. For BrdU incorporation, 49 day-old mice were treated with intraperitoneal injection of $50 \mathrm{mg} / \mathrm{Kg}$ of $\mathrm{BrdU}$ (Sigma-Aldrich, St Louis, MO), every 2 hours $\times 5$, then sacrificed 2 hours later. 4-8 $\mu \mathrm{m}$ sections were cut from paraffin-embedded tissues and deparaffinized. Antigen retrieval was performed in a microwave at high power for 5 minutes, followed by low power for 5 minutes $\mathrm{x} 2$ in citrate antigen retrieval buffer $(\mathrm{pH}$ 6.0). Slides were incubated with anti-Ki67 (NovoCastra, Newcastle, UK), anti-pH2AX (Cell Signaling Technology, Danvers, MA), anti-Dec1, anti-DcR2, anti-MnSOD, anti-p15Ink4b, or anti-Cdk2 antibodies(Santa Cruz Biotechnology), followed by biotinylated secondary antibody; and detected using streptavidin conjugated to horseradish peroxidase and DAB substrate (DAKO, Carpinteria, CA). For immunofluorescence staining, anti-H3K9me3 (Upstate Laboratories, Syracuse, NY), anti-4HNE (Alpha Diagnostic, San Antonio, TX, USA), anti-BrdU, antip21 (Santa Cruz Biotechnology), anti-phosphorylated Chk1 at Ser345 (Cell Signaling Technology), anti-14-33 , and anti-8(OH)dG (Abcam, Cambridge, UK) antibodies were detected with Cyanine 2, Cyanine 3, or Alexafluor488 secondary antibodies. The number of Ki67 positive cells, pH2AX positive cells, and BrdUpositive cells was manually counted from 5-7 representative fields, at 200x magnification, and normalized to total cell number. Digital photomicrographs were obtained using a Zeiss 510 NLO multiphoton/ confocal laser scanning microscope. Composite images were constructed using Photoshop CS4 software (Adobe Systems, Mountain View, CA). 


\section{Cell Explantation and ex-vivo culture}

Pineal cells were explanted at postnatal day 10 (P10); tumors were explanted when clinically apparent (bulging cranium). Cells were plated onto 8-well permanox chamber slides (Nunc, Rochester, NY), and cultured in DMEM with $10 \%$ FBS, $1 \%$ glutamine, and $1 \%$ Pen/Strep.

\section{DCFDA Assay}

To measure intracellular ROS in vitro, cells were treated with a peroxide sensitive reagent CM-H2DCF-DA (CMH2DCF-DA; Molecular Probes, Eugene, OR) at $10 \mu \mathrm{M}$ for $20 \mathrm{~min}$ at $37^{\circ} \mathrm{C}$ and observed under a fluorescence microscope.

\section{$\mathrm{N}$-Acetyl Cysteine treatment}

Explanted pineal cells were treated with N-Acetyl Cysteine (Sigma Aldrich) at a concentration of $5 \mathrm{mM}$. Media was renewed daily. Cells were treated for 10 days, and stained for SABG as described [20]. For DDR pathway analysis, cells were fixed and stained after 4 days.

\section{CVT313 and NSC625987 treatment}

Explanted cells were treated with CVT313 at $5 \mu \mathrm{M}$ (Santa Cruz Biotechnology), NSC625987 at $1 \mu \mathrm{M}$ (Tocris Bioscience, Ellisville, MO, USA), or DMSO vehicle; media was renewed every 3 days. Cells were fixed and stained for SABG after 7 days, and counterstained with eosin. For quantification of proportion of cells positive for SABG, 10 random fields were selected, and digital photomicrographs were analyzed using Adobe Photoshop CS4 software, by color selection and area analysis. For quantification of cellular accumulation, all the area of the well was photographed over 12 fields. Digital photomicrographs were analyzed using Adobe Photoshop CS4 software, by area selection tool. For BrdU incorporation assay, cells were treated with BrdU at a concentration of $33 \mu \mathrm{M}$ for 2 hours, fixed with $50 \%$ methanol $/ 50 \%$ acetone solution for 2 minutes, then processed as detailed above.

\section{Additional files}

Additional file 1: Figure S1: A) High-magnification images of senescence-associated heterochromatin foci (SAHF) marked by H3K9me3, in Irbp-Cyclin D1 (D1) versus wild-type (WT) pineal cells at P49. B) Senescence-associated beta galatosidase $(S A B G)$ staining of cultured pineal cells explanted from wild-type (WT), Irbp-Cyclin D1 (D1), and IrbpCyclin D1, p53 - - (D1p53-/-) animals, as indicated. SABG staining was done after 10 days of culture. C) Top panel: BrdU-incorporation and immunofluorescence staining of wild-type (WT) and Irbp-Cyclin D1 (D1) pineal cells, as indicated, after 10 days in culture. Bottom panel: corresponding DAPI-stained nuclei. D) Right panels: Immunofluorescence staining for $8(\mathrm{OH}) \mathrm{dG}$ and pH2AX, in wild-type (WT), Irbp-Cyclin D1 (D1), and NAC-treated Irbp-Cyclin D1 explanted pineal cells, as indicated. Left panels: corresponding DAPI staining of nuclei.

Additional file 2: Figure S2: A) Representative immunohistochemical staining for Ki67 (left) and immunoflourescence for H3K9me3 (right) and corresponding DAPI (middle) staining of Irbp-Cyclin D1, p18lnk4C-/- pineal sections at the indicated ages. B) Immunostaining for Dec1 and DcR2, in Irbp-Cyclin D1, p18/nk4c-/- pineal glands at the indicated ages. C) SABG staining of cultured Irbp-Cyclin D1, p18Ink4C -/- pineal cells after 14 and 20 days in culture, as indicated. D) Western blotting for the indicated proteins in pineal glands of the indicated genotypes, at the indicated ages.

\section{Conflict of interest}

The authors declare that they have no competing interests in relation to the work described.

\section{Author's contributions}

$\mathrm{HZ}$ carried out the most of the cellular and molecular studies, and participated in drafting the manuscript. MH, LM, and NEC performed immunohistochemical studies, mouse genotyping for the reported experiments, and helped in drafting the manuscript. GD and SXS participated in the design of the study and reviewed the manuscript. RS conceived the study, and participated in its design and coordination and drafted the manuscript. All authors read and approved the final manuscript.

\section{Acknowledgments}

This work was supported by an MPP grant from the American University of Beirut, and in part by the American Lebanese Syrian Associated Charities (ALSAC) and International Outreach Program at St Jude Children's Research Hospital, Memphis, TN. The authors thank Bristol-Myers Squibb, M. Barbacid, and M. F. Roussel for donating the p181nk4C - - mice; and support from the AUB Core Laboratory Shared Resources.

\section{Author details}

'Department of Pediatric and Adolescent Medicine, American University of Beirut, Beirut, Lebanon. ${ }^{2}$ Children's Cancer Center of Lebanon, American University of Beirut, Beirut, Lebanon. ${ }^{3}$ Pediatric Hematology-Oncology, University of Chicago, Chicago, Illinois, USA.

Received: 2 January 2012 Accepted: 10 April 2012 Published: 1 May 2012

\section{References}

1. Campisi J: d'Adda di Fagagna F: Cellular senescence: when bad things happen to good cells. Nat Rev Mol Cell Biol 2007, 8:729-740.

2. Saab R: Cellular senescence: many roads, one final destination. ScientificWorldJournal 2010, 10:727-741.

3. Beauséjour CM, Krtolica A, Galimi F, Narita M, Lowe SW, Yaswen P, Campisi J: Reversal of human cellular senescence: roles of the p53 and p16 pathways. EMBO J 2003, 22:4212-4222.

4. Zhu J, Woods D, McMahon M, Bishop JM: Senescence of human fibroblasts induced by oncogenic Raf. Genes Dev 1998, 12:2997-3007.

5. Harvey M, Sands AT, Weiss RS, Hegi ME, Wiseman RW, Pantazis P, Giovanella BC, Tainsky MA, Bradley A, Donehower LA: In vitro growth characteristics of embryo fibroblasts isolated from p53-deficient mice. Oncogene 1993, 8:2457-2467.

6. Sage J, Mulligan GJ, Attardi LD, Miller A, Chen S, Williams B, Theodorou E, Jacks T: Targeted disruption of the three Rb-related genes leads to loss of G(1) control and immortalization. Genes Dev 2000, 14:3037-3050.

7. Braig M, Lee $S$, Loddenkemper C, Rudolph C, Peters AH, Schlegelberger B, Stein H, Dörken B, Jenuwein T, Schmitt CA: Oncogene-induced senescence as an initial barrier in lymphoma development. Nature 2005, 436:660-665.

8. Herbig U, Jobling WA, Chen BP, Chen DJ, Sedivy JM: Telomere shortening triggers senescence of human cells through a pathway involving ATM, p53, and p21(CIP1), but not p16(INK4a). Mol Cell 2004, 14:501-513.

9. Takahashi A, Ohtani N, Yamakoshi K, lida S, Tahara H, Nakayama K, Nakayama Kl, Ide T, Saya H, Hara E: Mitogenic signalling and the p16INK4a-Rb pathway cooperate to enforce irreversible cellular senescence. Nat Cell Biol 2006, 8:1291-1297.

10. Mallette FA, Goumard S, Gaumont-Leclerc MF, Moiseeva O, Ferbeyre G: Human fibroblasts require the Rb family of tumor suppressors, but not p53, for PML-induced senescence. Oncogene 2004, 23:91-99.

11. Campaner S, Doni M, Hydbring P, Verrecchia A, Bianchi L, Sardella D, Schleker T, Perna D, Tronnersjö S, Murga M, et al: Cdk2 suppresses cellular 
senescence induced by the c-myc oncogene. Nat Cell Biol 2010, 12:54-59. sup pp 51-14

12. Hydbring P, Bahram F, Su Y, Tronnersjö S, Högstrand K, von der Lehr N Sharifi HR, Lilischkis R, Hein N, Wu S, et al: Phosphorylation by Cdk2 is required for Myc to repress Ras-induced senescence in cotransformation. Proc Natl Acad Sci U S A 2010, 107:58-63.

13. Saab R, Rodriguez-Galindo C, Matmati K, Rehg JE, Baumer SH, Khoury JD, Billups C, Neale G, Helton KJ, Skapek SX: p18Ink4c and p53 Act as tumor suppressors in cyclin D1-driven primitive neuroectodermal tumor. Cancer Res 2009, 69:440-448.

14. Lazzerini Denchi E, Attwooll C, Pasini D, Helin K: Deregulated E2F activity induces hyperplasia and senescence-like features in the mouse pituitary gland. Mol Cell Biol 2005, 25:2660-2672.

15. Sarkisian CJ, Keister BA, Stairs DB, Boxer RB, Moody SE, Chodosh LA: Dose-dependent oncogene-induced senescence in vivo and its evasion during mammary tumorigenesis. Nat Cell Bio/ 2007, 9:493-505.

16. Young AP, Schlisio S, Minamishima YA, Zhang Q, Li L, Grisanzio C, Signoretti S, Kaelin WG: VHL loss actuates a HIF-independent senescence programme mediated by $\mathrm{Rb}$ and p400. Nat Cell Biol 2008, 10:361-369.

17. Kennedy AL, McBryan T, Enders GH, Johnson FB, Zhang R, Adams PD: Senescent mouse cells fail to overtly regulate the HIRA histone chaperone and do not form robust Senescence Associated Heterochromatin Foci. Cell Div 2010, 5:16.

18. Guenatri M, Bailly D, Maison C, Almouzni G: Mouse centric and pericentric satellite repeats form distinct functional heterochromatin. J Cell Biol 2004, 166:493-505.

19. Maison C, Bailly D, Peters AH, Quivy JP, Roche D, Taddei A, Lachner M, Jenuwein T, Almouzni G: Higher-order structure in pericentric heterochromatin involves a distinct pattern of histone modification and an RNA component. Nat Genet 2002, 30:329-334.

20. Dimri GP, Lee X, Basile G, Acosta M, Scott G, Roskelley C, Medrano EE, Linskens M, Rubelj I, Pereira-Smith O: A biomarker that identifies senescent human cells in culture and in aging skin in vivo. Proc Natl Acad Sci U S A 1995, 92:9363-9367.

21. Collado M, Gil J, Efeyan A, Guerra C, Schuhmacher AJ, Barradas M, Benguría A, Zaballos A, Flores JM, Barbacid M, et al: Tumour biology: senescence in premalignant tumours. Nature 2005, 436:642.

22. Bartek J, Bartkova J, Lukas J: DNA damage signalling guards against activated oncogenes and tumour progression. Oncogene 2007 26:7773-7779.

23. Irani K, Xia Y, Zweier JL, Sollott SJ, Der CJ, Fearon ER, Sundaresan M, Finkel T, Goldschmidt-Clermont PJ: Mitogenic signaling mediated by oxidants in Ras-transformed fibroblasts. Science 1997, 275:1649-1652.

24. Lee AC, Fenster BE, Ito H, Takeda K, Bae NS, Hirai T, Yu ZX, Ferrans VJ, Howard BH, Finkel T: Ras proteins induce senescence by altering the intracellular levels of reactive oxygen species. J Biol Chem 1999, 274:7936-7940

25. Moiseeva O, Bourdeau V, Roux A, Deschênes-Simard X, Ferbeyre G: Mitochondrial dysfunction contributes to oncogene-induced senescence. Mol Cell Biol 2009, 29:4495-4507.

26. Li Z, Shi K, Guan L, Cao T, Jiang Q, Yang Y, Xu C: ROS leads to MnSOD upregulation through ERK2 translocation and p53 activation in selenite-induced apoptosis of NB4 cells. FEBS Lett 2010, 584:2291-2297.

27. Sherr CJ, Roberts JM: Inhibitors of mammalian G1 cyclin-dependent kinases. Genes Dev 1995, 9:1149-1163.

28. Uziel T, Zindy F, Sherr CJ, Roussel MF: The CDK inhibitor p18Ink4c is a tumor suppressor in medulloblastoma. Cell Cycle 2006, 5:363-365.

29. Nakayama K, Ishida N, Shirane M, Inomata A, Inoue T, Shishido N, Horii I, Loh DY: Mice lacking p27(Kip1) display increased body size, multiple organ hyperplasia, retinal dysplasia, and pituitary tumors. Cell 1996, 85:707-720.

30. Krimpenfort $P$, ljpenberg A, Song JY, van der Valk M, Nawijn M, Zevenhoven $\mathrm{J}$, Berns A: p15Ink4b is a critical tumour suppressor in the absence of p16Ink4a. Nature 2007, 448:943-946.

31. Brooks EE, Gray NS, Joly A, Kerwar SS, Lum R, Mackman RL, Norman TC, Rosete J, Rowe M, Schow SR, et al: CVT-313, a specific and potent inhibitor of CDK2 that prevents neointimal proliferation. J Biol Chem 1997, 272:29207-29211.

32. Kubo A, Nakagawa K, Varma RK, Conrad NK, Cheng JQ, Lee WC, Testa JR, Johnson BE, Kaye FJ, Kelley MJ: The p16 status of tumor cell lines identifies small molecule inhibitors specific for cyclin-dependent kinase 4. Clin Cancer Res 1999, 5:4279-4286.
33. Mclnnes C, Wang S, Anderson S, O'Boyle J, Jackson W, Kontopidis G, Meades C, Mezna M, Thomas M, Wood G, et al: Structural determinants of CDK4 inhibition and design of selective ATP competitive inhibitors. Chem Biol 2004, 11:525-534.

34. Braig M, Schmitt CA: Oncogene-induced senescence: putting the brakes on tumor development. Cancer Res 2006, 66:2881-2884.

35. Narita M, Nũnez S, Heard E, Lin AW, Hearn SA, Spector DL, Hannon GJ, Lowe $\mathrm{SW}$ : Rb-mediated heterochromatin formation and silencing of E2F target genes during cellular senescence. Cell 2003, 113:703-716.

36. Ye X, Zerlanko B, Zhang R, Somaiah N, Lipinski M, Salomoni P, Adams PD: Definition of pRB- and p53-dependent and -independent steps in HIRA/ASF1a-mediated formation of senescence-associated heterochromatin foci. Mol Cell Biol 2007, 27:2452-2465.

37. Chan HM, Narita M, Lowe SW, Livingston DM: The p400 E1A-associated protein is a novel component of the p53 ->p21 senescence pathway. Genes Dev 2005, 19:196-201.

38. Guan $\mathrm{KL}$, Jenkins CW, Li Y, Nichols MA, Wu X, O'Keefe CL, Matera AG, Xiong Y: Growth suppression by $\mathrm{p} 18$, a p16INK4/MTS1- and p14INK4B/MTS2-related CDK6 inhibitor, correlates with wild-type pRb function. Genes Dev 1994, 8:2939-2952.

39. Hirai H, Roussel MF, Kato JY, Ashmun RA, Sherr CJ: Novel INK4 proteins, p19 and p18, are specific inhibitors of the cyclin D-dependent kinases CDK4 and CDK6. Mol Cell Biol 1995, 15:2672-2681.

40. Sotillo R, Renner O, Dubus P, Ruiz-Cabello J, Martín-Caballero J, Barbacid M, Carnero A, Malumbres M: Cooperation between Cdk4 and p27kip1 in tumor development: a preclinical model to evaluate cell cycle inhibitors with therapeutic activity. Cancer Res 2005, 65:3846-3852.

41. Skapek SX, Lin SC, Jablonski MM, McKeller RN, Tan M, Hu N, Lee EY: Persistent expression of cyclin D1 disrupts normal photoreceptor differentiation and retina development. Oncogene 2001, 20:6742-6751.

42. Latres E, Malumbres M, Sotillo R, Martín J, Ortega S, Martín-Caballero J, Flores JM, Cordón-Cardo C, Barbacid M: Limited overlapping roles of P15 (INK4b) and P18(INK4c) cell cycle inhibitors in proliferation and tumorigenesis. EMBO J 2000, 19:3496-3506.

doi:10.1186/1476-4598-11-28

Cite this article as: Zalzali et al.: Temporally distinct roles for tumor suppressor pathways in cell cycle arrest and cellular senescence in Cyclin D1-driven tumor. Molecular Cancer 2012 11:28.

\section{Submit your next manuscript to BioMed Central and take full advantage of:}

- Convenient online submission

- Thorough peer review

- No space constraints or color figure charges

- Immediate publication on acceptance

- Inclusion in PubMed, CAS, Scopus and Google Scholar

- Research which is freely available for redistribution 